\title{
On the Fekete-Szegö Problem for a Class of Analytic Functions
}

\author{
Zhigang Peng \\ Department of Mathematics, Hubei University, Wuhan 430062, China \\ Correspondence should be addressed to Zhigang Peng; pengzhigang@hubu.edu.cn
}

Received 2 December 2013; Accepted 21 January 2014; Published 4 March 2014

Academic Editors: Y. Han and B. Jacob

Copyright (C) 2014 Zhigang Peng. This is an open access article distributed under the Creative Commons Attribution License, which permits unrestricted use, distribution, and reproduction in any medium, provided the original work is properly cited.

Let $\mathscr{A}$ denote the class of functions which are analytic in the unit disk $D=\{z:|z|<1\}$ and given by the power series $f(z)=$ $z+\sum_{n=2}^{\infty} a_{n} z^{n}$. Let $C$ be the class of convex functions. In this paper, we give the upper bounds of $\left|a_{3}-\mu a_{2}^{2}\right|$ for all real number $\mu$ and for any $f(z)$ in the family $\mathscr{V}=\{f(z): f \in \mathscr{A}, \operatorname{Re}(f(z) / g(z))>0$ for some $g \in C\}$.

\section{Introduction}

Let $\mathscr{A}$ denote the class of functions which are analytic in the unit disk $D=\{z:|z|<1\}$ and satisfy $f(0)=f^{\prime}(0)-$ $1=0$. The set of all functions $f \in \mathscr{A}$ that are univalent will be denoted by $S$. Let $C, S^{*}(\beta)$ and $K$ be the classes of convex, starlike of order $\beta$ and close-to-convex functions, respectively. Fekete and Szegö [1] proved that

$$
\left|a_{3}-\mu a_{2}^{2}\right| \leq \begin{cases}4 \mu-3, & \mu \geq 1, \\ 1+2 \exp \left[\frac{-2 \mu}{(1-\mu)}\right], & 0 \leq \mu<1, \\ 3-4 \mu, & \mu<0\end{cases}
$$

holds for any $f(z)=z+\sum_{n=2}^{\infty} a_{n} z^{n} \in S$ and that this inequality is sharp. The coefficient functional $\Lambda_{\mu}(f)=a_{3}-\mu a_{2}^{2}$ on $f$ in $\mathscr{A}$ plays an important role in function theory. For example, $a_{3}-a_{2}^{2}=S_{f}(0) / 6$, where $S_{f}$ is the Schwarzian derivative. The problem of maximizing the absolute value of the functional $\Lambda_{\mu}(f)$ is called the Fekete-Szegö problem. In the literature, there exist a large number of results about the Fekete-Szegö problem (see, for instance, [2-11]).

For $0 \leq \alpha<1$ and $0 \leq \beta<1$, let $u_{\alpha}^{\beta}$ denote the class of functions $f$ satisfying $f \in \mathscr{A}$ and

$$
\operatorname{Re}\left\{\frac{\alpha z^{2} f^{\prime \prime}(z)}{g(z)}+\frac{z f^{\prime}(z)}{g(z)}\right\}>0
$$

for some $g \in S^{*}(\beta)$. Al-Abbadi and Darus [7] investigated the Fekete-Szegö problem on the class $u_{\alpha}^{\beta}$.
Let $C_{1}(\beta)$ be the class of functions $f$ in $\mathscr{A}$ satisfying the inequality

$$
\operatorname{Re}\left\{\frac{z f^{\prime}(z)}{\phi(z)} e^{i \beta}\right\}>0 \quad\left(|z|<1,-\frac{\pi}{2}<\beta<\frac{\pi}{2}\right)
$$

for some function $\phi \in C$. In [11], Srivastara et al. studied the Fekete-Szegö problem on the class

$$
C_{1}=\bigcup_{\beta} C_{1}(\beta) \quad\left(-\frac{\pi}{2}<\beta<\frac{\pi}{2}\right)
$$

for $0 \leq \mu \leq 1$ by proving that

$$
\left|a_{3}-\mu a_{2}^{2}\right| \leq \begin{cases}\frac{5}{3}-\frac{9}{4} \mu, & 0 \leq \mu \leq \frac{2}{9}, \\ \frac{2}{3}+\frac{1}{9 \mu}, & \frac{2}{9} \leq \mu \leq \frac{2}{3}, \\ \frac{5}{6}, & \frac{2}{3}<\mu \leq 1 .\end{cases}
$$

Srivastara et al. held that the inequality (5) was sharp. However, the extremal function given in [11] did not exist in the case of $2 / 3<\mu \leq 1$.

In this paper, we solve the Fekete-Szegö problem for the family

$$
\mathscr{V}=\left\{f(z): f \in \mathscr{A}, \operatorname{Re} \frac{f(z)}{g(z)}>0 \text { for some } g \in C\right\} .
$$


As a corollary of the main result, we find the sharp upper bounds for absolute value of the Fekete-Szegö functional for the class $v_{\alpha}$ defined by

$$
\begin{aligned}
& v_{\alpha}=\left\{f(z): f \in \mathscr{A}, \operatorname{Re}\left\{\frac{\alpha z^{2} f^{\prime \prime}(z)}{g(z)}+\frac{z f^{\prime}(z)}{g(z)}\right\}>0\right. \\
& \text { for some } g \in C\} .
\end{aligned}
$$

Clearly, $v_{\alpha}$ is a subclass of $u_{\alpha}^{0}$. In the case of $\alpha=0$, we get sharp estimation of the absolute value of the Fekete-Szegö functional for the class $C_{1}(0)$ and for all real number $\mu$, which prove that the inequality (5) is not sharp actually when $2 / 3<$ $\mu \leq 1$.

\section{Main Result}

Let $B_{0}$ be the class of functions $\phi(z)$ that are analytic in $D$ and satisfy $|\phi(z)| \leq|z|$ for all $|z|<1$. The following two lemmas can be found in [2].

Lemma 1 (see [2]). If $\phi(z)=\sum_{n=1}^{\infty} \alpha_{n} z^{n}$ is in the class $B_{0}$, then, for any complex number $s$, one has $\left|\alpha_{2}-s \alpha_{1}^{2}\right| \leq 1+(|s|-$ 1) $\left|\alpha_{1}^{2}\right| \leq \max \{1,|s|\}$. The inequality is sharp.

Lemma 2 (see [2]). If $g(z)=z+\sum_{n=2}^{\infty} c_{n} z^{n}$ is in the class $C$ and $\mu$ is a complex number, then $\left|c_{3}-\mu c_{2}^{2}\right| \leq 1 / 3+(|\mu-1|-$ $1 / 3)\left|c_{2}^{2}\right| \leq \max \{1 / 3,|\mu-1|\}$. The inequality is sharp.

Theorem 3. If $f(z)=z+\sum_{n=2}^{\infty} a_{n} z^{n}$ is in the class $\mathscr{V}$ and $\mu$ is a real number, then

$$
\left|a_{3}-\mu a_{2}^{2}\right| \leq \begin{cases}5-9 \mu, & \text { when } \mu \leq \frac{1}{6}, \\ 2+\frac{1}{4 \mu}, & \text { when } \frac{1}{6} \leq \mu \leq \frac{1}{2}, \\ 3-2 \mu+\frac{1}{4(1-\mu)}, & \text { when } \frac{1}{2}<\mu \leq \frac{5}{6} \\ 7 \mu-3, & \text { when } \frac{5}{6}<\mu \leq 1, \\ 9 \mu-5, & \text { when } 1 \leq \mu .\end{cases}
$$

Proof. By definition, $f(z)=z+\sum_{n=2}^{\infty} a_{n} z^{n}$ is in the class $\mathscr{V}$ if and only if there exists a function $g(z)=z+\sum_{n=2}^{\infty} c_{n} z^{n} \in C$ such that $\phi(z)=[f(z)-g(z)] /[f(z)+g(z)]=\sum_{n=1}^{\infty} \alpha_{n} z^{n} \epsilon$ $B_{0}$. A simple computation shows $a_{2}=c_{2}+2 \alpha_{1}, a_{3}=c_{3}+$ $2\left(\alpha_{1} c_{2}+\alpha_{2}+\alpha_{1}^{2}\right)$. Thus,

$a_{3}-\mu a_{2}^{2}=c_{3}-\mu c_{2}^{2}+2\left[\alpha_{2}+(1-2 \mu) \alpha_{1}^{2}\right]+2(1-2 \mu) \alpha_{1} c_{2}$.
So, by Lemmas 1 and 2, we have

$$
\begin{aligned}
\mid a_{3}- & \mu a_{2}^{2} \mid \\
\leq & \left|c_{3}-\mu c_{2}^{2}\right|+2\left|\alpha_{2}+(1-2 \mu) \alpha_{1}^{2}\right| \\
& +2\left|1-2 \mu\left\|\alpha_{1}\right\| c_{2}\right| \\
\leq & \frac{1}{3}+\left[|\mu-1|-\frac{1}{3}\right]\left|c_{2}\right|^{2}+2\left[1+(|1-2 \mu|-1)\left|\alpha_{1}\right|^{2}\right] \\
& +2\left|1-2 \mu\left\|\alpha_{1}\right\| c_{2}\right| .
\end{aligned}
$$

Putting $\left|\alpha_{1}\right|=x$ and $\left|c_{2}\right|=y$, we get from (10) that $\left|a_{3}-\mu a_{2}^{2}\right| \leq$ $F(x, y)$, where

$$
\begin{aligned}
F(x, y)= & \frac{1}{3}+\left[|\mu-1|-\frac{1}{3}\right] y^{2} \\
& +2\left[1+(|1-2 \mu|-1) x^{2}\right] \\
& +2|1-2 \mu| x y .
\end{aligned}
$$

Since $\left|\alpha_{1}\right| \leq 1$ and $\left|c_{2}\right| \leq 1$, we will calculate the maximum value of $F(x, y)$ for $(x, y) \in[0,1] \times[0,1]$.

Case 1. Suppose $\mu \leq 1 / 2$. Then it follows from (11) that

$$
F(x, y)=\frac{7}{3}+\left(\frac{2}{3}-\mu\right) y^{2}-4 \mu x^{2}+2(1-2 \mu) x y .
$$

Since

$$
\begin{gathered}
F_{x}(x, y)=-8 \mu x+2(1-2 \mu) y, \\
F_{y}(x, y)=2(1-2 \mu) x+\left(\frac{4}{3}-2 \mu\right) y, \\
\left|\begin{array}{cc}
-8 \mu & 2(1-2 \mu) \\
2(1-2 \mu) & \frac{4}{3}-2 \mu
\end{array}\right|=\frac{16}{3} \mu-4<0,
\end{gathered}
$$

$F(x, y)$ does not have a local maximum at any point of the open rectangle $(0,1) \times(0,1)$. Hence, $F(x, y)$ must attain its maximum at a boundary point. Since $F(0, y) \leq 3-$ $\mu, F(1, y) \leq F(1,1)=5-9 \mu$ for $\mu \leq 1 / 2$ and

$$
F(x, 0) \leq \begin{cases}\frac{7}{3}-4 \mu, & \text { if } \mu<0 \\ \frac{7}{3}, & \text { if } 0 \leq \mu \leq \frac{1}{2},\end{cases}
$$

$$
F(x, 1) \leq \begin{cases}F(1,1)=5-9 \mu, & \text { if } \mu<\frac{1}{6}, \\ F\left(\frac{1-2 \mu}{4 \mu}, 1\right)=\frac{1}{4 \mu}+2, & \text { if } \frac{1}{6} \leq \mu \leq \frac{1}{2},\end{cases}
$$

we have

$$
\left|a_{3}-\mu a_{2}^{2}\right| \leq F(x, y) \leq \begin{cases}5-9 \mu, & \text { when } \mu \leq \frac{1}{6}, \\ 2+\frac{1}{4 \mu}, & \text { when } \frac{1}{6}<\mu \leq \frac{1}{2} .\end{cases}
$$


Case 2. Suppose $1 / 2<\mu \leq 1$. Then, we get from (11) that

$$
F(x, y)=\frac{7}{3}+\left(\frac{2}{3}-\mu\right) y^{2}+4(\mu-1) x^{2}+2(2 \mu-1) x y .
$$

Since

$$
\begin{gathered}
F_{x}(x, y)=8(\mu-1) x+2(2 \mu-1) y, \\
F_{y}(x, y)=2(2 \mu-1) x+\left(\frac{4}{3}-2 \mu\right) y, \\
\left|\begin{array}{cc}
8(\mu-1) & 2(2 \mu-1) \\
2(2 \mu-1) & \frac{4}{3}-2 \mu
\end{array}\right|=4\left(-8 \mu^{2}+\frac{32}{3} \mu-\frac{11}{3}\right)<0,
\end{gathered}
$$

$F(x, y)$ must attain its maximum at a boundary point of the rectangle $[0,1] \times[0,1]$.

Since

$$
\begin{gathered}
F(x, 0) \leq \frac{7}{3}, \quad \text { if } \frac{1}{2}<\mu \leq 1, \\
F(x, 1) \leq\left\{\begin{array}{l}
F\left(\frac{1-2 \mu}{4(\mu-1)}, 1\right) \\
=3-2 \mu+\frac{1}{4(1-\mu)}, \quad \text { if } \frac{1}{2}<\mu \leq \frac{5}{6}, \\
F(1,1)=7 \mu-3,
\end{array}\right. \\
F(0, y) \leq\left\{\begin{array}{l}
3-\mu, \quad \text { if } \frac{1}{2}<\mu \leq \frac{2}{6}, \\
\frac{7}{3},
\end{array} \quad \text { if } \frac{2}{3}<\mu \leq 1,\right. \\
F(1, y) \leq F(1,1)=7 \mu-3, \quad \text { if } \frac{1}{2}<\mu \leq 1,
\end{gathered}
$$

we get

$$
\begin{aligned}
\left|a_{3}-\mu a_{2}^{2}\right| & \leq F(x, y) \\
& \leq \begin{cases}3-2 \mu+\frac{1}{4(1-\mu)}, & \text { when } \frac{1}{2}<\mu \leq \frac{5}{6}, \\
7 \mu-3, & \text { when } \frac{5}{6}<\mu \leq 1 .\end{cases}
\end{aligned}
$$

Case 3. Suppose $\mu>1$. Then, (11) gives

$$
F(x, y)=\frac{7}{3}+\left(\mu-\frac{4}{3}\right) y^{2}+4(\mu-1) x^{2}+2(2 \mu-1) x y .
$$

Since

$$
\begin{gathered}
F_{x}(x, y)=8(\mu-1) x+2(2 \mu-1) y, \\
F_{y}(x, y)=2(2 \mu-1) x+\left(2 \mu-\frac{8}{3}\right) y, \\
\left|\begin{array}{cc}
8(\mu-1) & 2(2 \mu-1) \\
2(2 \mu-1) & 2 \mu-\frac{8}{3}
\end{array}\right|=\frac{4}{3}(13-16 \mu)<0,
\end{gathered}
$$

$F(x, y)$ must attain its maximum at a boundary point of the rectangle $[0,1] \times[0,1]$. Since

$$
\begin{aligned}
& F(x, 0) \leq 4 \mu-\frac{5}{3}, \quad \text { if } \mu \geq 1, \\
& F(x, 1) \leq F(1,1)=9 \mu-5, \quad \text { if } \mu \geq 1, \\
& F(0, y) \leq \begin{cases}\frac{7}{3}, & \text { if } 1<\mu \leq \frac{4}{3}, \\
1+\mu, & \text { if } \mu>\frac{4}{3},\end{cases} \\
& F(1, y) \leq F(1,1)=9 \mu-5, \quad \text { if } \mu \geq 1,
\end{aligned}
$$

it follows that

$$
\left|a_{3}-\mu a_{2}^{2}\right| \leq F(x, y) \leq 9 \mu-5, \quad \text { when } 1<\mu \text {. }
$$

Combining (15), (19) with (23), we get (8). Since inequalities in Lemmas 1 and 2 are sharp, it follows that inequality (8) is also sharp. The proof is completed.

Since $f \in v_{\alpha}$ if and only if $F(z)=\alpha z^{2} f^{\prime \prime}(z)+z f^{\prime}(z) \in \mathscr{V}$, by a simple calculation, we have the following.

Corollary 4. If $f(z)=z+\sum_{n=2}^{\infty} a_{n} z^{n} \in v_{\alpha}$, then

$$
\begin{aligned}
& \left|a_{3}-\mu a_{2}^{2}\right|
\end{aligned}
$$

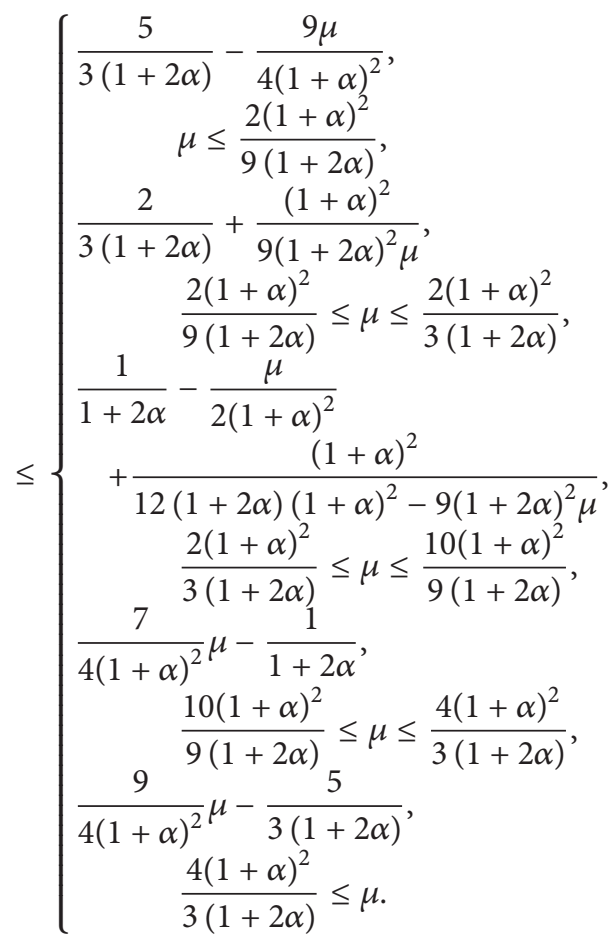


Corollary 5. If $f(z)=z+\sum_{n=2}^{\infty} a_{n} z^{n} \in C_{1}(0)$, then

$$
\begin{aligned}
& \left|a_{3}-\mu a_{2}^{2}\right| \\
& \leq \begin{cases}\frac{5}{3}-\frac{9}{4} \mu, & \text { when } \mu \leq \frac{2}{9}, \\
\frac{2}{3}+\frac{1}{9 \mu}, & \text { when } \frac{2}{9} \leq \mu \leq \frac{2}{3}, \\
1-\frac{1}{2} \mu+\frac{1}{12-9 \mu}, & \text { when } \frac{2}{3}<\mu \leq \frac{10}{9}, \\
\frac{7}{4} \mu-1, & \text { when } \frac{10}{9}<\mu \leq \frac{4}{3}, \\
\frac{9}{4} \mu-\frac{5}{3}, & \text { when } \frac{4}{3} \leq \mu .\end{cases}
\end{aligned}
$$

Remark 6. In [11], Srivastava et al. gave a function $f(z)=z+$ $\sum_{n=2}^{\infty} a_{n} z^{n} \in \mathscr{A}$ satisfying $\phi(z)=[f(z)-g(z)] /[f(z)+g(z)]=$ $\sum_{n=1}^{\infty} \alpha_{n} z^{n} \in B_{0}$, where $g(z)=z+z^{2}+z^{3}+\cdots \in C, \alpha_{2}=1-\alpha_{1}^{2}$ and

$$
\alpha_{1}=\frac{(2-3 \lambda) \pm i \sqrt{6 \mu-4}}{6 \mu} .
$$

Srivastava held that $f \in C_{1}(0)$ and $\left|a_{3}-\mu a_{2}^{2}\right|=5 / 6$ when $2 / 3 \leq \mu \leq 1$. But $\phi \in B_{0}$ implies that $\left|\alpha_{2}\right| \leq 1-\left|\alpha_{1}^{2}\right|$. So $\phi(z)$ satisfying the above conditions does not exist.

\section{Conflict of Interests}

The author declares that there is no conflict of interests regarding the publication of this paper.

\section{Acknowledgment}

The paper is supported by Educational Commission of Hubei Province of China (D2011006).

\section{References}

[1] M. Fekete and G. Szegö, "Eine bemerkung über ungerade schlichte funktionen," The Journal of the London Mathematical Society, vol. 8, no. 2, pp. 85-89, 1933.

[2] F. R. Keogh and E. P. Merkes, "A coefficient inequality for certain classes of analytic functions," Proceedings of the American Mathematical Society, vol. 20, pp. 8-12, 1969.

[3] H. R. Abdel-Gawad and D. K. Thomas, "The Fekete-Szegö problem for strongly close-to-convex functions," Proceedings of the American Mathematical Society, vol. 114, no. 2, pp. 345-349, 1992.

[4] R. R. London, "Fekete-Szegö inequalities for close-to-convex functions," Proceedings of the American Mathematical Society, vol. 117, no. 4, pp. 947-950, 1993.

[5] N. E. Cho, "On the Fekete-Szegö problem and argument inequality for strongly quasi-convex functions," Bulletin of the Korean Mathematical Society, vol. 38, no. 2, pp. 357-367, 2001.

[6] S. Kanas and H. E. Darwish, "Fekete-Szegö problem for starlike and convex functions of complex order," Applied Mathematics Letters, vol. 23, no. 7, pp. 777-782, 2010.
[7] M. H. Al-Abbadi and M. Darus, "The Fekete-Szegö theorem for a certain class of analytic functions," Sains Malaysiana, vol. 40, no. 4, pp. 385-389, 2011.

[8] H. Orhan, E. Deniz, and D. Raducanu, "The Fekete-Szegö problem for subclasses of analytic functions defined by a differential operator related to conic domains," Computers \& Mathematics with Applications, vol. 59, no. 1, pp. 283-295, 2010.

[9] K. Al-Shaqsi and M. Darus, "On Fekete-Szegö problems for certain subclass of analytic functions," Applied Mathematical Sciences, vol. 2, no. 9-12, pp. 431-441, 2008.

[10] B. Bhowmik, S. Ponnusamy, and K.-J. Wirths, "On the FeketeSzegö problem for concave univalent functions," Journal of Mathematical Analysis and Applications, vol. 373, no. 2, pp. 432438, 2011.

[11] H. M. Srivastava, A. K. Mishra, and M. K. Das, "The FeketeSzegö problem for a subclass of close-to-convex functions," Complex Variables and Elliptic Equations, vol. 44, no. 2, pp. 145163, 2001. 


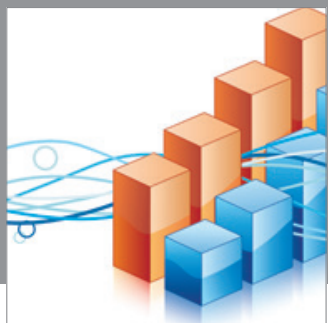

Advances in

Operations Research

mansans

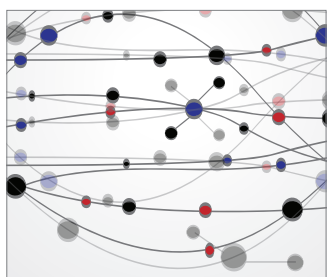

The Scientific World Journal
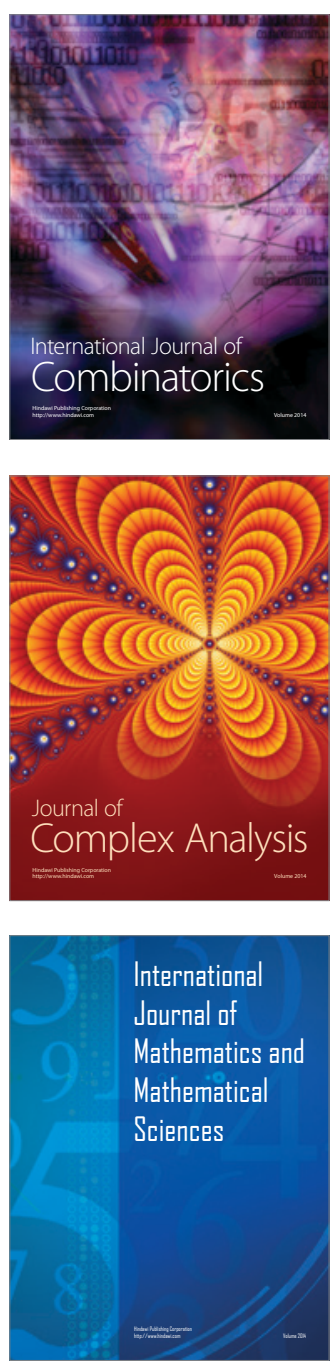
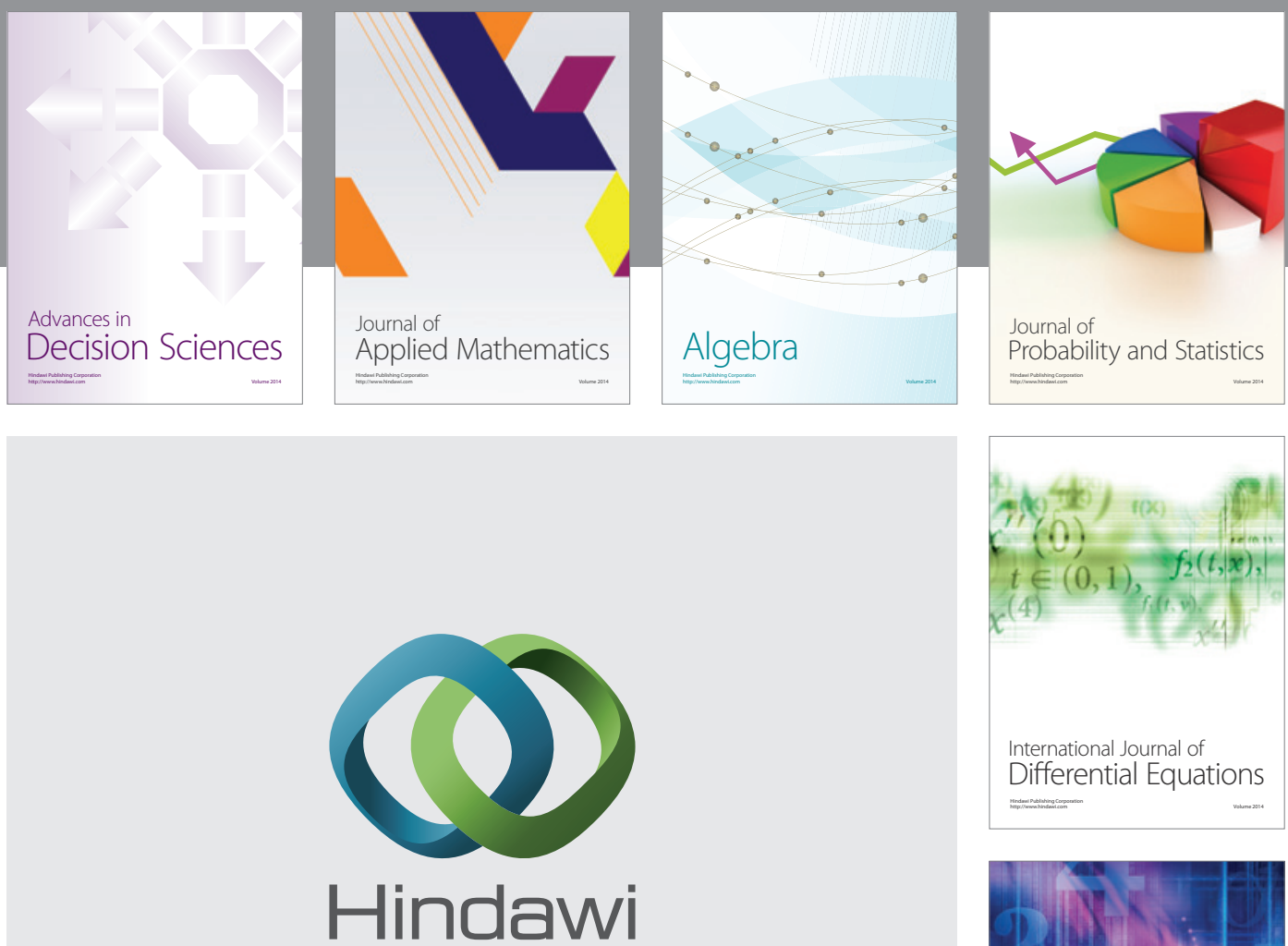

Submit your manuscripts at http://www.hindawi.com
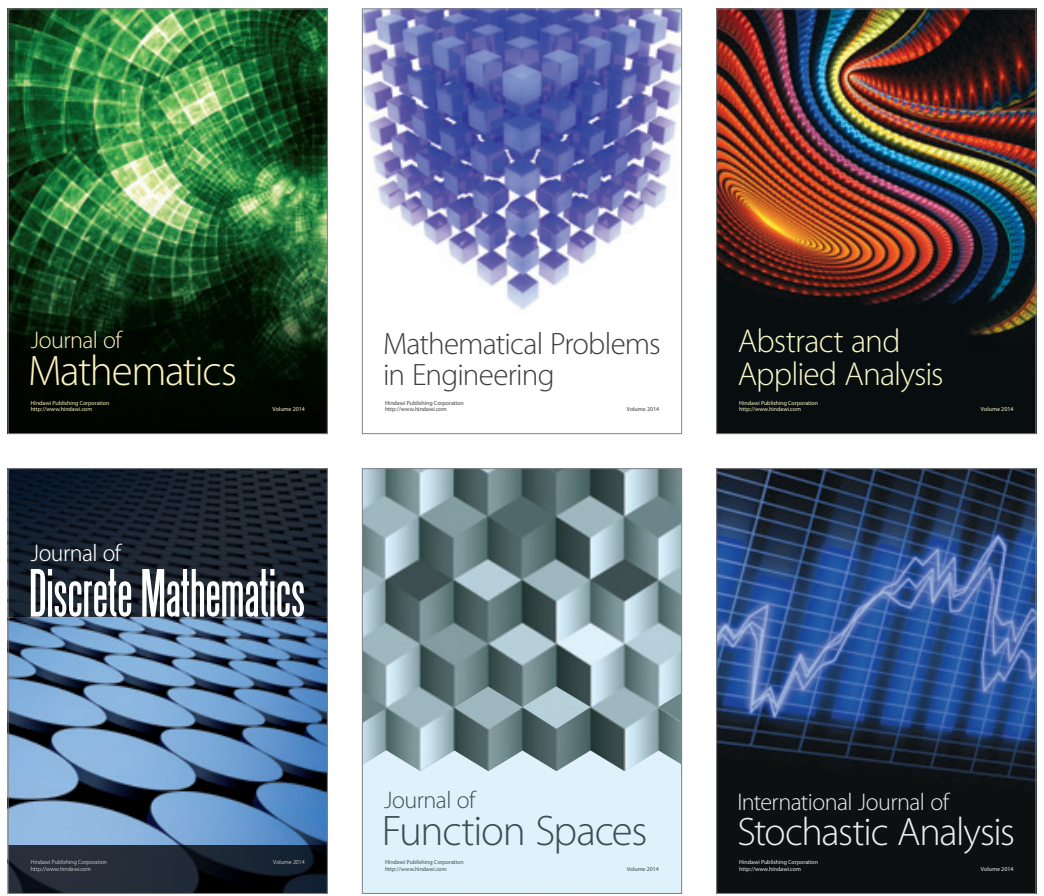

Journal of

Function Spaces

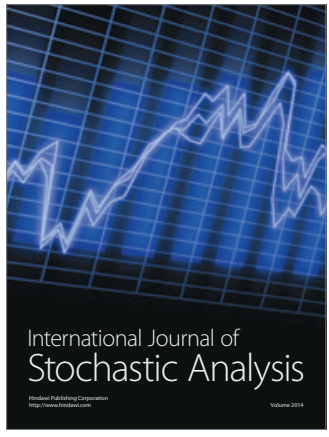

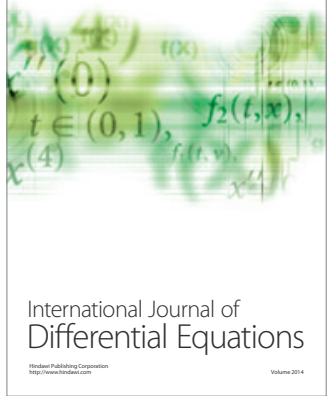
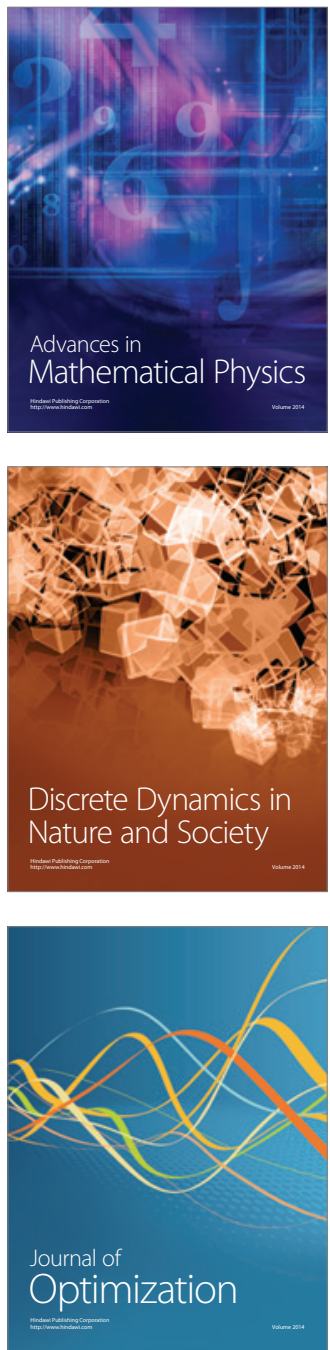